\title{
Assessing the Accuracy of Land-Based Mobile Laser Scanning Data
}

\section{Introduction}

Recent years have been marked by a development of many different surveying techniques using laser scanners, among which mobile laser scanning systems (MLS) are promising. Mobile scanning system is a complex system and its development using modular way depends on the needs. The quality of 3D data strongly depends on the exact determination of the position as well as the orientation of the laser scanner sensor during the data acquisition process. Furthermore, the navigational solution is the largest possible source of error in a MLS [8]. Different obstructions of GNSS (Global Navigation Satellite Systems) reception appearing in urban or sub-urban areas diminish the accuracy of position and attitude information significantly. Errors of position resulting from poor satellite geometry, as well as frequent expected gaps in GNSS signals directly influence the performance of vehicle-based laser scanning system [1]. This type of disruptions impacts all the geometry of the collected point cloud. The continuity of localization can be compensated by the inertial unit. However, a unique characteristic of INS (Inertial Navigation System) is that it provides a high degree of accuracy only temporary because it gradually degrades over time. This is caused by three main sources of error: initial alignment errors for roll, pitch and yaw angles, inertial sensor errors (e.g. gyroscope drift, accelerometer shift) and computational errors [10].

In this paper a prototype mobile platform designed and developed at the laboratory of MINES ParisTech was described. In the following section a method for assessing the accuracy of mobile laser scanning data is presented. The methodology adopted by the study was to validate the received data, rather than assess the individual components of the system. To ensure real evaluation, a reference data of an accuracy of at least an order of magnitude better than the studied system has been used.

* AGH University of Science and Technology, Faculty of Mining Surveying and Environmental Engineering, Krakow, Poland; MINES ParisTech, CAOR Centre de Robotique, Mathématique et Systèmes, Paris, France

** MINES ParisTech, CAOR Centre de Robotique, Mathématique et Systèmes, Paris, France 


\section{Validation of Laser Data}

The aim of qualification is to classify point cloud data, whether the result is acceptable or not. Basically there are two approaches that enable the qualification of objects from the database and evaluate their quality. They assess quality using coefficients (qualitative method), by figures (quantitative analysis), or a mix of both [4]. Quantitative data analysis, known also as absolute, often contains descriptive statistics and inferential statistics. The performance of MLS may be assessed in terms of resolution, accuracy and repeatability for the captured data [5]. In this approach, quantitative resolution of point clouds is defined in terms of point and profile spacing. At the same time, the latter of the defined criteria (repeatability) demands from the system to be capable of producing data of equal quality on each occasion.

The validation methods used nowadays are mostly based on accuracy assessment. A review of the literature reveals some examples of reports on such validation work. As an example [6] presents the method of quality evaluation of laser point clouds consisting in positioning accuracy analysis by comparing coordinates of two differently collected sets of points and analysis of their spatial distribution. To evaluate the accuracy, they propose using the control points in the form of newly designed 3D target points spaced on the experimental field (vertical wall) and measured by the total station. However, [7] and [2] report the use of Real Time Kinematic (RTK) GPS to provide a consistent set against which the performance of the mobile laser system could be evaluated. Assessing the planimetric and elevation accuracy of a vehicle-based laser scanning system is here conducted by comparing the collected laser data against the check points collected at corner points of white line markings. Another assessment of overall error of the 3D point cloud collected by the aforementioned system is performed by [9] choosing the reference data of surfaces selected semi automatically from the available 3D city model. In order to assess the accuracy of laser data, planar surfaces were estimated by least squares adjustment, which then were compared with the given façade polygon from the building model.

\section{Details of the LARA 3D Platform}

A prototype of a vehicle-based laser scanning system named LARA3D has been developed by the Robotics Laboratory (CAOR) of MINES ParisTech since 2003. In the present configuration of LARA3D the system is equipped with: one GPS receiver (a single frequency AgGPS132 from Trimble), one strap down Inertial Measurement Unit (either Crossbow VG600 or Crossbow IMU 440) and two odometers for the left and right wheels as localization sensors. Our perception sensors consist of two SICK LMS 221 laser scanners with a field of view of $180^{\circ}$ placed back-to-back and two cameras Canon EOS 5D with fisheye lens (Fig. 1). 


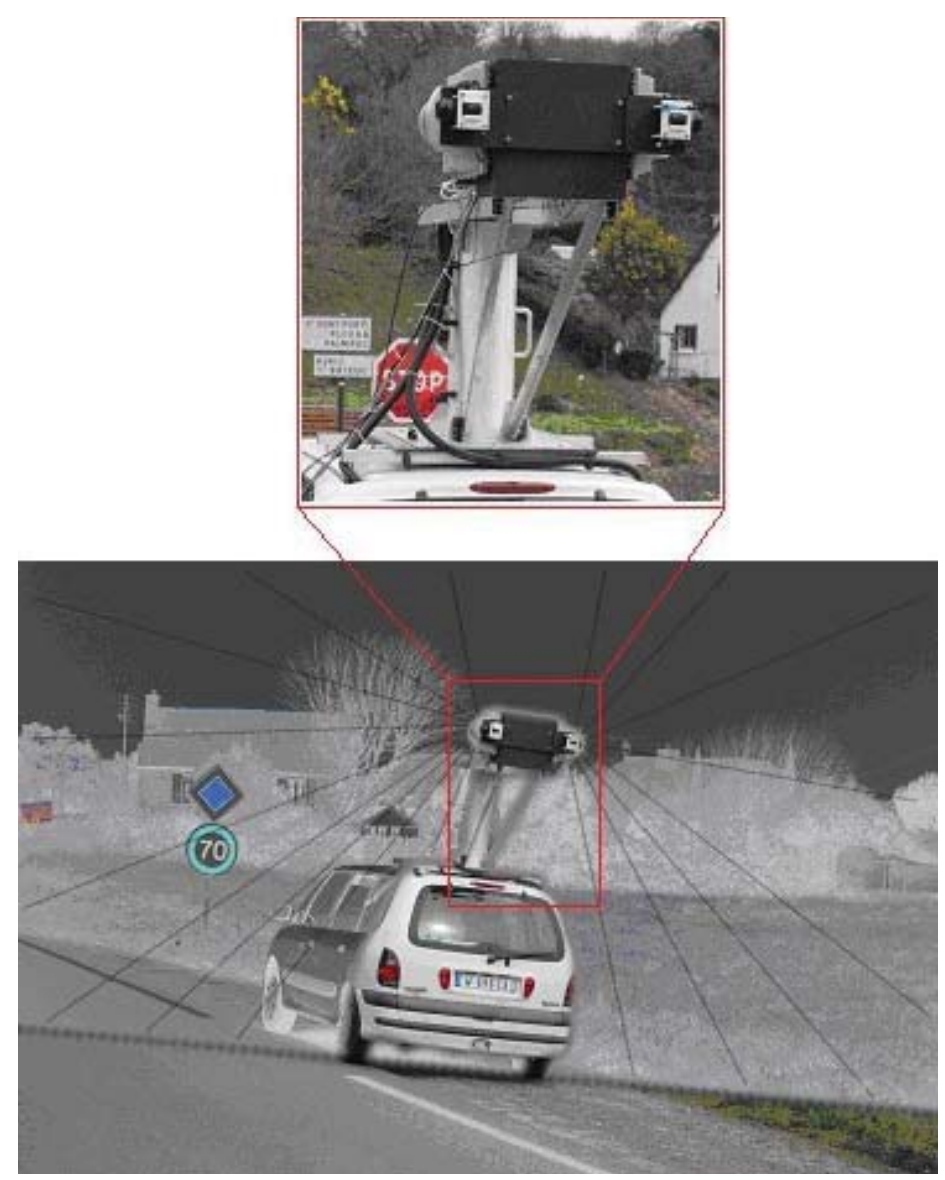

Fig. 1. The LARA 3D prototype (version 2009)

MLS system components are mounted on a Renault Espace van, whereas mapping sensors (scanners and cameras) are placed at a height of $3.1 \mathrm{~m}$ above ground. The Lara3D is a system which integrates the localization sensors using a loosely coupled INS feedback scheme with a typical 15 state Extended Kalman Filter. For this purpose, we use an environment called RTMaps ${ }^{\circledR}$ which enables us to register the data in real time using specifically written software components.

\section{Accuracy Analysis}

The mobile mapping system selected for the study is our LARA3D system, in the configuration described above. In order to assess its accuracy, reference measurements were required. This phase consists in the comparison with data collected using another more accurate approach. Laser point cloud quality collected by our 
mobile system was examined by comparison with a classical survey performed with a Total Station. Alternatively to this measurement a 3D point cloud collected by static laser scanner was also used as a reference. This provided two independent datasets enabling assessment of mobile data using two completely different approaches. The main interest of our investigations was the evaluation of data quality for points at building façades as it is a product of mobile scanning frequently used for generating photo-realistic 3D models. The urban acquisitions were conducted in Paris near the Musée d'Orsay. This is typical urban location that can be characterized by compact, high development and narrow streets (Fig. 2).

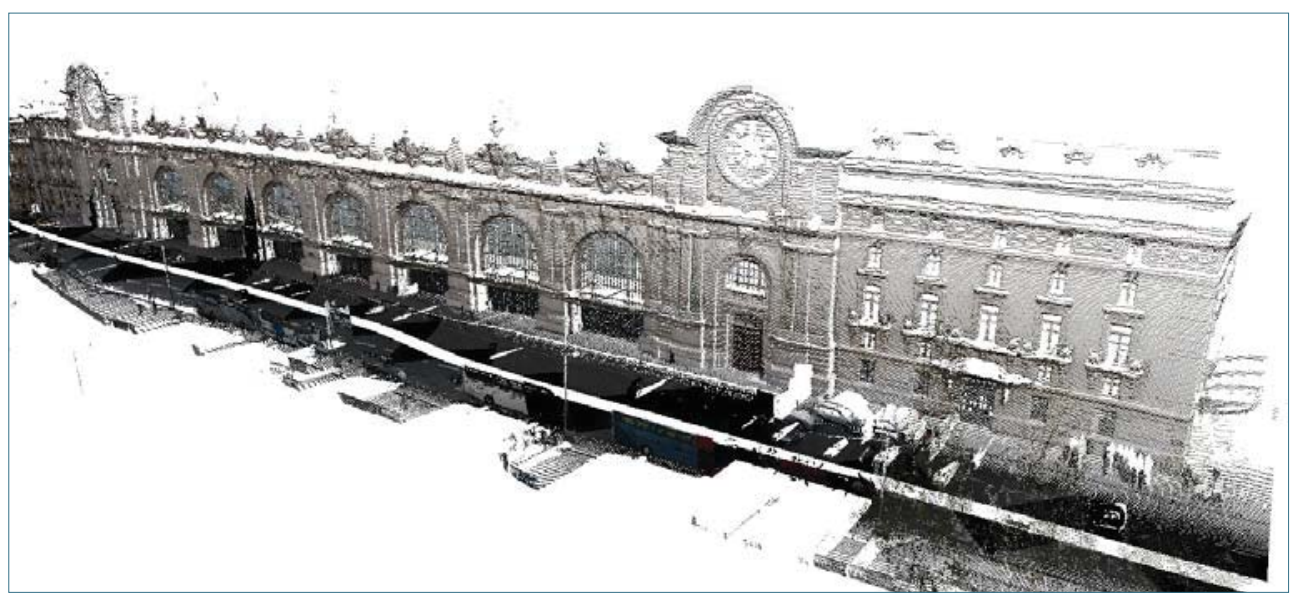

Fig. 2. MLS point cloud - northern façade of Musée d'Orsay

\subsection{Reference Data}

We have gathered two independent sets of reference data. The first of them included coordinates of points - architectural details (not signalled by means of targets), mostly building horns and edges of plinths and pillars present in the Northern façade of Musée d'Orsay. These elements, found as well recognized in point cloud data, were measured using classical methods with Leica TCR803 Prismless Total Station. Instrument positions have been localized along the façade at distances closer than $30 \mathrm{~m}$. In total, 344 points were collected, 47 of which are target points located uniformly along the wall, the remaining belonging to the profile intersecting the building at an approx. height of $7.2 \mathrm{~m}$ above street level (Fig. 3).

The second set of reference data was a terrestrial laser point cloud. Measurements were made combining Trimble ${ }^{\circledR} G X$ - pulse based laser scanner and Trimble®VX Spatial Station - phase based scanner. In the case of Musée d'Orsay, the main target of scanning were two perpendicular façades (Northern and Western). The 26 scans were made from different positions: 10 for Trimble ${ }^{\circledR} G X$ scanner and 16 Trimble®VX scanner. 


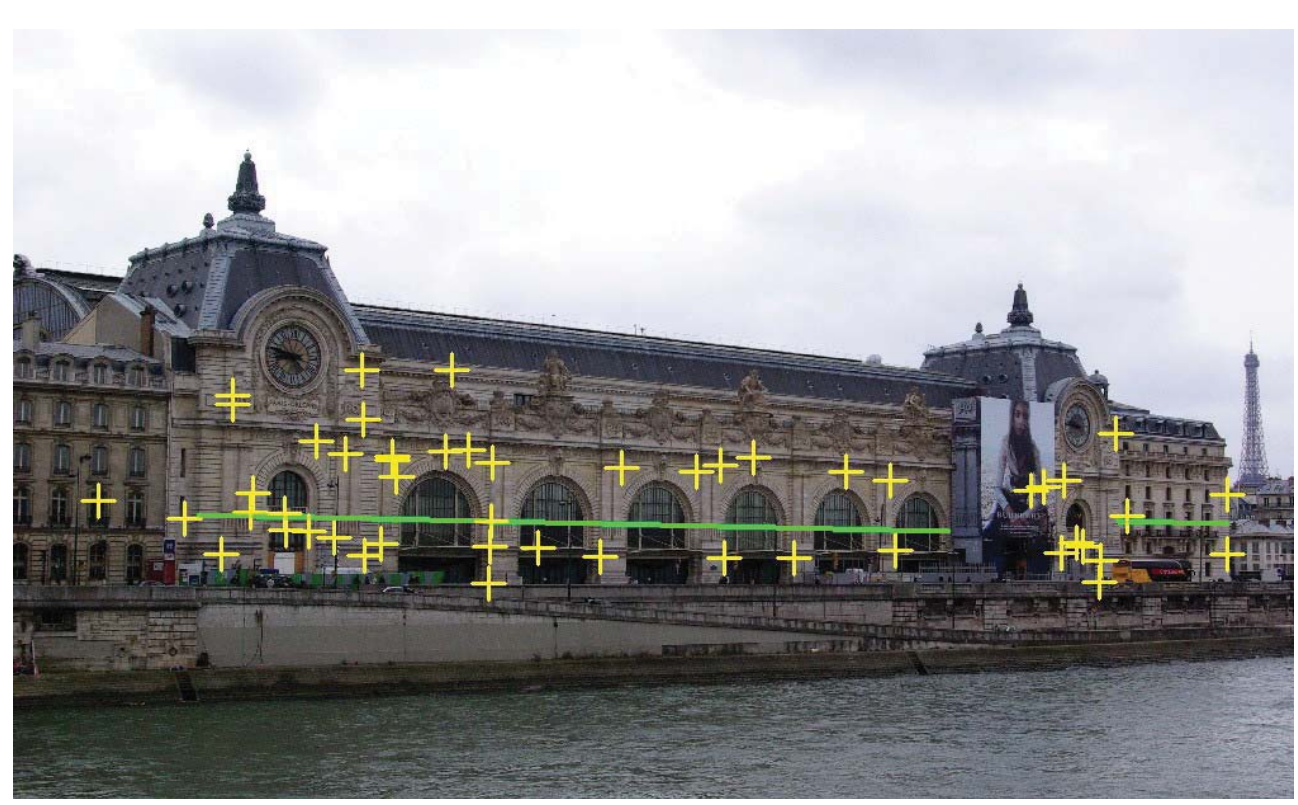

Fig. 3. Control points (yellow crosses) used as a reference data. Profile course shown in green

Both total station surveys as well as TLS point cloud data were treated as local data, not connected to the National survey grid. Thus, the entire study includes only "relative" analyses. Points collected using total station were measured with accuracy about $1 \mathrm{~cm}$. The final point cloud density adopted as the reference cloud was reduced to $2 \mathrm{~cm}$.

\subsection{Comparison with Total Station Survey}

For this comparison, all points located in the different areas of façades have been used in order to calculate 43 sections connecting the selected details between each other in different configurations. The corresponding sections were also found and manually measured in the "mobile" point cloud. These operations were performed for the directly georeferenced point cloud, positioned by the integration of the INS/GPS/Odometer data using RTMaps ${ }^{\circledR}$ software. Figure 4 shows that point cloud accuracy is similar and for 35\% of the control sections taken for examinations, the difference in length between the controlled section and the reference does not exceed $0.1 \mathrm{~m}$, and for $79 \% 0.2 \mathrm{~m}$.

It should be emphasized that the weakest point of this method was the necessity to manually indicate the points in the MLS cloud that correspond to the details. A man-made error is always present during the extraction of the key points (whether or not signalled) and it strongly depends on the point cloud resolution. On the other hand, there is still strong need to automatize the whole process. 


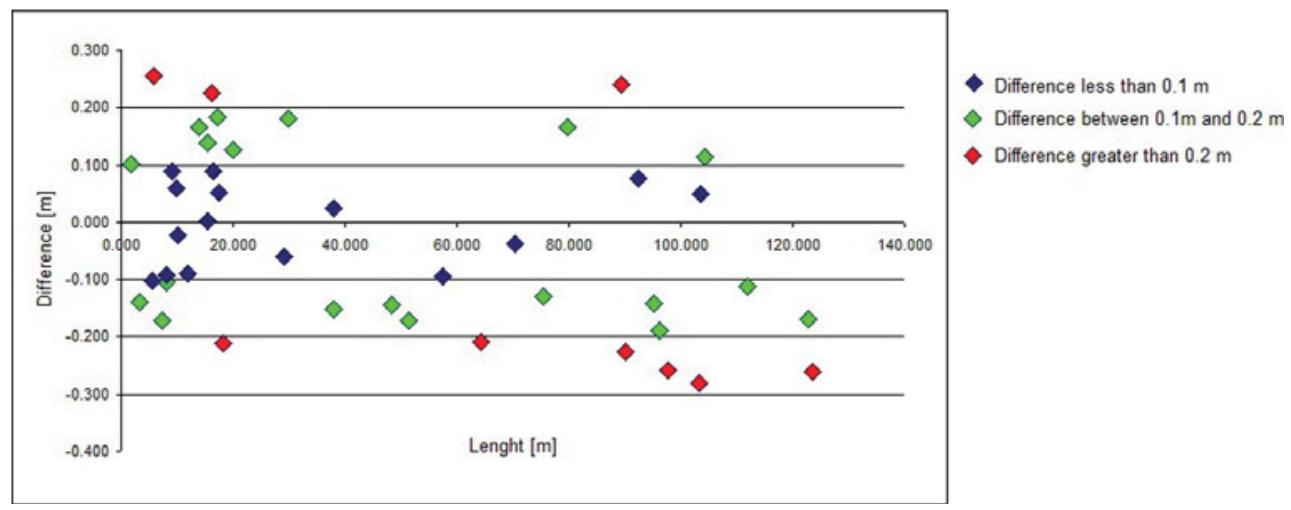

Fig. 4. Diagram of differences [m] of control section lengths

Afterwards, the quality of our point cloud was visually checked with relation to the section measured using Total Station. As shown below (Fig. 5), our mobile point cloud reveals some local deformations (occasionally is locally stretched or shifted). The reason for this is most probably the imperfection of the geo-referencing i.e result of Kalman observer algorithm and of sensor noise models.

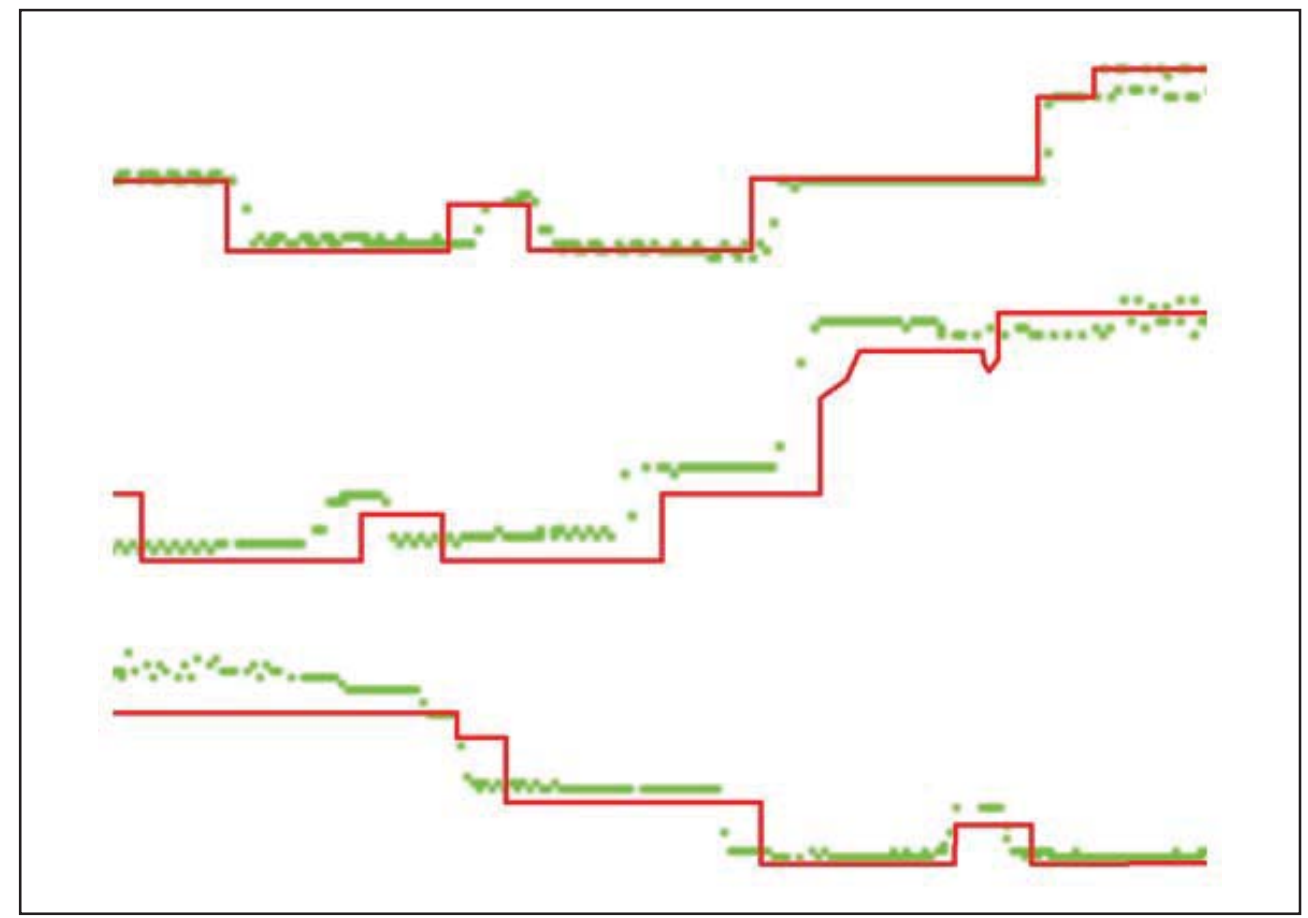

Fig. 5. Selected cross-section from the classical survey (in red) with corresponding profile from mobile point cloud (in green) 


\subsection{Geometric Matching MLS Data with Static TLS}

Originally introduced by Besl McKay [3], the ICP (Iterative Closest Point) algorithm aims to find the transformation between point cloud and some reference surface (or another point cloud), by minimizing the square errors between corresponding points. An important application of this algorithm is to register actual data sensed from 3D object with an ideal 3D model.

To investigate the accuracy of mobile data, matching of corresponding MLS point clouds based on ICP algorithm was made. Considering the fact that mobile point clouds can be degraded by the temporary bad quality of system positioning, an approach of the evaluation was conducted in two ways. In the first one, registration for the whole mobile point cloud was made. As a result the average distances of $0.185 \mathrm{~m}$ between "mobile" and reference static laser point clouds was obtained. In this case, the result in the form of the shift between the surfaces derive from errors in the mobile LiDAR measurements rather than man-made errors. To verify the correctness of the achieved result, a further analysis based on the cross-section was performed for 4 levels located on different heights. A significant degradation of MLS point cloud quality increasing with the altitude of building façade was noticeable. Manual measurements of distances between both laser point clouds showed that these values vary from $0 \mathrm{~m}$ to $0.285 \mathrm{~m}$. At the same time, it has been noticed that the part of mobile point cloud was highly locally deformed because of very poor quality of signal received by the AgGPS132 (Trimble) receiver. Afterwards, the MLS point cloud of Musée d'Orsay was divided into 6 blocks. The registration of each fragment with the corresponding reference cloud was made separately, and values for the average distance have been determined (in Tab. 1).

Table 1. Matching results for each piece of point cloud

\begin{tabular}{||l|c|c||}
\hline \multicolumn{1}{|c||}{ Name of Dataset } & Distance $[\mathrm{m}]$ & Mean [m] \\
\hline \hline DataSet_part1* & - & \\
\cline { 1 - 2 } DataSet_part2 & 0.042 & \multirow{2}{*}{0.060} \\
\cline { 1 - 2 } DataSet_part3 & 0.063 & \\
\cline { 1 - 2 } DataSet_part4 & 0.064 & \\
\cline { 1 - 2 } DataSet_part5 & 0.071 & \\
\hline DataSet_part6 & 0.058 & \\
\hline
\end{tabular}

* Fragment of point cloud affected by very poor GPS quality

In this approach, it can be noticed that the average distance of mobile point clouds from the reference clouds has improved. Such a result proves that the point cloud collected by LARA3D is locally deformed. Insightful analysis of DataSet_part1 confirmed that proper matching was not possible for the mentioned surface. The obtained result for this part was not consistent with the reality. 


\section{Conclusion}

In this paper, we have described an accuracy analysis of MLS point clouds collected using the LARA3D prototype platform in an urban area. Accuracy of the MLS was achieved through comparison with other data sources more accurate that the studied system. The study has shown, that when compared with control points, collected by a Total Station, the prototype system LARA3D is able to produce data with an accuracy better then $0.3 \mathrm{~m}$. However, taking into consideration the uncertainty in the identification of common points, this method is affected by man-made error and limited by point cloud resolutions.

Meanwhile, the use of existing reference data, such as e.g. high resolution point clouds from static terrestrial laser scanning provides fast and reliable data evaluation. The subjective element of operator interpretation is also removed. Results achieved using ICP algorithm show, that our mobile mapping system suffers from limitations of the sensor quality and Kalman filter implementation. In the case of point clouds locally degraded, proper matching is impossible and the obtained result does not reflect the type and scale of deformation correctly.

Meanwhile, another less time-consuming and more automated method for assessing data accuracy should be developed. That may be referred to using the existing spatial data as reference, such as e.g.: cadastre, ALS data, Topographic Data Base (TBD), Digital Terrain Model, orthophotos and so on.

\section{References}

[1] Abuhadrous I.: Système embarqué temps réel de localisation et de modélisation 3D par fusion multi-capteur. MINES ParisTech, 2005, pp. 38-41.

[2] Barber D., Mills J., Smith-Voysey S.: Geometric validation of a ground-based mobile laser scanning system. ISPRS Journal of Photogrammetry \& Remote Sensing, vol. 63, 2008, pp. 128-141.

[3] Besl P.J., McKay N.D.: A method for registration of 3-D shapes. IEEE Transactions on Pattern Analysis and Machine Intelligence, vol. 14, no. 2, pp. 239-256.

[4] Boulaassal H.: Segmentation et modélisation géométrique de façades de bâtiments à partir de relevés laser terrestres. Université de Strasbourg 2010, pp. 125-163.

[5] Cahalane C.: Research presented in poster founded by IRCSET. Pavement Management Services and Strategic Research Cluster Grant "Assessing Mobile Mapping System Performance", [on-line:] www.stratag.ie/content/projects/ posters/CCahalane_Final.ppt.

[6] Feng J., Zhong R., Yang Y., Zhao W.: Quality Evaluation of Spatial Point-Cloud Data Collected by Vehicle-Borne Laser Scanner. International Workshop on Education and Training \& International Workshop on Geosciences and Remote Sensing, 2008. 
[7] Gandofi S., Barbarella M., Ronci E., Bruchi A.: Close photogrammetry and laser scanning using a mobile mapping system for high detailed survey of a high density urban area. The International Archives of the Photogrammetry, Remote Sensing and Spatial Information Sciences, vol. XXXVII, part B5, 2008, pp. 909-914.

[8] Glennie C.: Rigorous 3D error analysis of kinematic scanning LIDAR systems. Journal of Applied Geodesy, vol. 1, 2007, pp. 147-157.

[9] Haala N., Peter M., Kremer J., Hunter G.: Mobile Lidar Mapping for 3D point cloud collection in urban areas - a performance test. XXI ISPRS Congress, 2008.

[10] Titterton D.H.: Strapdown Inertial Navigation Technology (2nd Edition). The Institution of Electrical Engineering, 2004, pp. 335-375. 